\title{
Acute Motor and Sensory Axonal Neuropathy
}

National Cancer Institute

\section{Source}

National Cancer Institute. Acute Motor and Sensory Axonal Neuropathy. NCI Thesaurus.

Code C116927.

A subtype of Guillain-Barre syndrome that targets sensory motor axons, and is characterized by acute onset of quadriparesis, distal sensory loss, areflexia, and respiratory insufficiency. 